\title{
Avaliação dos aeroportos das cidades-sedes da Copa de 2014 através do método AHP
}

\author{
Helen Feuser Fernandes ${ }^{1}$, Hadassa Pereira de Carvalho², \\ Cláudio Jorge Pinto Alves ${ }^{3}$ e Anderson Ribeiro Correia ${ }^{4}$
}

\begin{abstract}
Resumo: Esse estudo busca apresentar um método de classificação dos aeroportos das cidades-sedes da Copa de 2014, de acordo com critérios prioritários na opinião dos passageiros. O intuito é contribuir com as autoridades aeroportuárias, a fim de identificar oportunidades de melhoria e gargalos operacionais. Para isso foram utilizados dois levantamentos de opinião dos passageiros: um desenvolvido pelo Ministério da Aviação Civil no Brasil em 2013, que foi aplicado em 12 aeroportos internacionais, e uma pesquisa desenvolvida em um grande aeroporto internacional no Brasil, que foi aplicado para obter os pesos de relevância de instalações. O Processo de Análise Hierárquica (AHP) foi utilizado para consolidar os resultados destas duas pesquisas, fornecendo um índice de qualidade global para cada um dos aeroportos pesquisados. Os resultados indicam que o Aeroporto de Curitiba possui a melhor infraestrutura dentre os aeroportos analisados, e que o Aeroporto de Manaus é o que necessita de mais adequações.
\end{abstract}

Palavras-chave: Aeroportos; AHP; Infraestrutura Aeroportuária.

\begin{abstract}
This paper aims to present a ranking method to assess the airports of the FIFA World Cup host cities, according to the priority in passenger opinion. The purpose is to contribute with the airport authorities, in order to identify the improvement opportunities as the operational bottlenecks. Two passenger surveys were used: a survey developed by the Ministry of Civil Aviation in Brazil in 2013, which was applied at 12 international airports and a survey developed at a major international airport in Brazil, which was applied to obtain the weights of relevance of facilities. The Analytic Hierarchy Process (AHP) was applied to consolidate the results of these two surveys, providing a global quality index for each surveyed airport. The results indicates that Curitiba Airport has the best infrastructure among the others analyzed airports and that Manaus Airport is that one who needs more adjustments. The results indicate that Curitiba Airport has the best infrastructure among all analyzed airports and that Manaus Airport is that one who needs more adjustments.
\end{abstract}

Keywords: Airports; AHP; Airport Infrastructure.

\section{INTRODUÇÃO}

O Brasil apresentou um grande crescimento econômico nos últimos anos, despontando no cenário mundial e, assim, conquistando o direito de sediar grandes eventos como a Jornada Mundial da Juventude de 2013, a Copa do Mundo de 2014 e os Jogos Olímpicos de 2016. Tendo em vista tais fatos, a SAC (Secretaria de Aviação Civil) deparou-se com a necessidade de obter a opinião dos passageiros sobre os serviços prestados nos principais aeroportos do país. Para tanto, foi aplicada uma pesquisa de campo, ao longo do ano de 2013, em um grupo de quinze aeroportos que estão diretamente relacionados a estes eventos, sendo os resultados divulgados trimestralmente.

Com o objetivo de apresentar um método para classificação dos aeroportos, este trabalho se propõe a aplicar a ferramenta AHP (Analytic Hierarchy Process) na avaliação dos aeroportos utilizados nos eventos da Copa do Mundo de

\footnotetext{
${ }^{1}$ Helen Feuser Fernandes, Departamento de Engenharia de Infraestrutura Aeronáutica. Instituto Tecnológico de Aeronáutica.

(helen.fernandes@gmail.com)

2 Hadassa Pereira de Carvalho, Departamento de Engenharia de

Infraestrutura Aeronáutica. Instituto Tecnológico de Aeronáutica.

(hadassa.pereira@gmail.com)

3 Cláudio Jorge Pinto Alves, Departamento de Engenharia de

Infraestrutura Aeronáutica. Instituto Tecnológico de Aeronáutica.

(claudioj@ita.br)

${ }^{4}$ Anderson Ribeiro Correia, Departamento de Engenharia de

Infraestrutura Aeronáutica. Instituto Tecnológico de Aeronáutica.

(correia@ita.br)
}

Manuscrito recebido em 17/06/2015 e aprovado para publicação em 15/09/2015.

Este artigo é parte de TRANSPORTES v. 23, n. 3, 2015. ISSN: 2237-1346 (online). DOI: $10.14295 /$ transportes.v23i3.952
2014. O estudo foi realizado com base nas informações colhidas no relatório da SAC (referente aos meses de abril a junho de 2013) e nos pesos de indicadores estabelecidos por Bandeira (2008). Assim, tem-se como contribuição a avaliação dos componentes aeroportuários de acordo com os pesos de importância que os usuários atribuem a esses componentes, diferentemente dos relatórios normalmente publicados que não levam em consideração a percepção dos passageiros. Diante deste cenário onde há a necessidade de avaliar vários requisitos e desenvolver avaliações complexas, pode-se recorrer ao método AHP. O qual apresenta uma metodologia de medição, que se baseia em comparações, par a par, feitas por meio do julgamento dos clientes a fim de gerar uma escala de prioridades (Saaty, 2008).

Segundo Fernandes e Pacheco (2007), quando o foco está na produtividade é preciso analisar os aspectos econômicos e operacionais a fim de proporcionar melhorias de ordem prática. Atualmente a dinâmica que rege os negócios exige uma grande flexibilidade no processo de gestão das organizações. Além disso, os cenários em que estão inseridas tornam-se cada vez mais complexos, de modo que diversos fatores internos e externos estão em constante mudança. Aeroportos são organizações que se encaixam perfeitamente ao cenário descrito pelos autores supracitados. Suas particularidades, tais como áreas comerciais, logística, segurança, e a sua importância para o desenvolvimento da sua região tornam seus processos e administração muito complexos e sujeitos à crítica de diversos tipos de clientes - de passageiros às companhias áreas.

Como a prestação de serviço tem um caráter qualitativo, há a necessidade de desenvolver indicadores de níveis 
Tabela 1. Lista dos aeroportos internacionais analisados

\begin{tabular}{ll}
\hline Sigla & \multicolumn{1}{c}{ AEROPORTOS INTERNACIONAIS } \\
ICAO & \multicolumn{1}{c}{ Aeroporto Internacional Juscelino Kubitschek - Brasília } \\
\hline SBBR & Aeron \\
SBCF & Aeroporto Internacional Tancredo Neves - Confins \\
SBCT & Aeroporto Internacional Afonso Pena - Curitiba \\
SBCY & Aeroporto Internacional Marechal Rondon - Cuiabá \\
SBEG & Aeroporto Internacional Eduardo Gomes - Manaus \\
SBFZ & Aeroporto Internacional Pinto Martins - Fortaleza \\
SBGL & Aeroporto Internacional Antônio Carlos Jobim/Galeão - Rio De Janeiro \\
SBGR & Aeroporto Internacional Gov. André Franco Montoro/Guarulhos - São Paulo \\
SBNT & Aeroporto Internacional Augusto Severo - Natal \\
SBPA & Aeroporto Internacional Salgado Filho - Porto Alegre \\
SBRF & Aeroporto Internacional Gilberto Freire - Recife \\
SBSV & Aeroporto Internacional Luiz Eduardo Magalhães - Salvador \\
\hline
\end{tabular}

de serviço para avaliar a satisfação dos passageiros em relação aos diversos aspectos do aeroporto. Muitos estudos já foram realizados neste contexto e com diferentes objetos de estudo, ora abordando o lado terra ou ar (Correia, Wirasinghe e Barros, 2007; Borille e Correia, 2013), ora atendo-se a um critério específico como segurança (Yoo e Choi, 2006). Além da criação destes indicadores é preciso encontrar ferramentas capazes de mensurá-los e de permitir identificar a importância relativa de cada um a fim de priorizar investimentos (Correia, Wirasinghe e Barros, 2007), garantir a satisfação dos clientes e comparar dois ou mais aeroportos de forma clara e estruturada.

Bezerra e Gomes (2015) desenvolveram um estudo com base em dados encontrados nos relatórios da SAC, no período de janeiro a dezembro de 2013, para o Aeroporto Internacional de Guarulhos. O objetivo era examinar a influência de determinados indicadores na satisfação dos passageiros, sendo esses: check-in, segurança, concessões, ambiente, instalações básicas, mobilidade e preços. Foi possível constatar que há maior probabilidade do passageiro atribuir uma classificação global mais elevada quanto maior for sua satisfação em relação ao check-in, segurança, ambiente, instalações gerais e preços. Sendo que o indicador "concessões” não demonstra possuir essa mesma influência (Bezerra e Gomes, 2015).

Da mesma forma, Chen et al. (2015), pesquisaram quais os fatores que mais influenciam na satisfação dos passageiros. Foram estudados três indicadores: acessibilidade, inspeção de segurança e instalações aeroportuárias, relacionadas com a satisfação do passageiro, e o valor para o cliente. A inspeção de segurança foi considerada como sendo o item mais importante na avaliação dos passageiros. Isso revela que haverá maior probabilidade da satisfação ser alcançada se houver melhorias na segurança. Houve também alta correlação entre a satisfação do cliente e o valor para o cliente, ou seja, quanto mais satisfeito o passageiro estiver, mais provavelmente ele estará disposto a utilizar o aeroporto.

Já com o mesmo intuito de comparar aeroportos entre si, Lupo (2015) estudou três aeroportos internacionais da região da Sicília (Itália), através de indicadores como: tempo de processamento, concessões, conforto, informações, funcionários e segurança. O autor também utilizou questionários para obter as respostas de passageiros e especialistas, e o auxílio da ferramenta AHP para ponderação dos critérios. Com esse trabalho foi possível indicar as fragilidades de cada aeroporto, e classificá-los de acordo com a qualidade de serviço entregue. Outros estudos ainda buscam estabelecer correlações entre o nível de qualidade de serviço percebida, e a rentabilidade do aeroporto, sendo um deles o trabalho de Merket e Assaf (2015). Os autores classificaram 33 aeroportos em relação à eficiência, para isso foram utilizadas proxies, que são variáveis que "explicam” de forma aproximada a realidade, sendo elas: número de passageiros, toneladas de carga e movimentação de aeronaves. Porém, através dos resultados fica evidenciado que a classificação dependerá do tipo de proxy escolhida, demonstrando as fortes características de cada aeroporto (Merket e Assaf, 2015).

Existe certa preocupação dos gestores aeroportuários e das companhias aéreas em relação à satisfação dos passageiros, porém, sabe-se da complexidade do sistema como um todo por haver inúmeros componentes envolvidos. Por isso, o estudo de avaliação de aeroportos toma relevância ao destacar as fragilidades, em especial em eventos extraordinários onde o sistema tenderá ao congestionamento, e os gargalos serão evidenciados.

Este trabalho está dividido da seguinte forma: na Seção 2 é descrita a metodologia aplicada; a Seção 3 abre a discussão da modelagem à luz dos resultados; por último, na conclusão são feitas as últimas ponderações e discussões em torno de todos os resultados do estudo aqui realizado.

\section{MÉTODO}

Para a avaliação dos aeroportos das cidades-sedes da Copa de 2014, tomou-se o relatório da SAC (2013) que contempla os meses de abril a junho de 2013. Este é resultante de uma pesquisa elaborada por especialistas, e aplicada aos usuários em diversos terminais de passageiros (entre os quais estão os aeroportos analisados neste trabalho). No total foram avaliados 15 aeroportos, dentre eles 13 são internacionais. No entanto, no estudo aqui realizado foram escolhidos apenas 12 dos aeroportos internacionais (Tabela 1) citados no relatório. Isto se deve ao fato de Campinas (o outro aeroporto internacional analisado pela SAC) se encontrar a uma distância significativa de uma das cidadessedes, aproximadamente 100 quilômetros de São Paulo.

Dos critérios avaliados pela SAC (2013), deu-se preferência àqueles referentes à infraestrutura dos terminais de passageiros, sendo esses: 
Tabela 2. Escala de prioridades dos critérios utilizados

\begin{tabular}{lc}
\hline Critérios & Prioridades \\
\hline Check-in & $1^{\circ}$ \\
Sala de embarque & $2^{\circ}$ \\
Acesso & $3^{\circ}$ \\
Saguão & $4^{\circ}$ \\
Concessões & $5^{\circ}$ \\
\hline
\end{tabular}

Tabela 3. Escala fundamental do método AHP de acordo com Saaty (1990)

\begin{tabular}{|c|c|c|}
\hline $\begin{array}{l}\text { Intensidade de } \\
\text { Importância }\end{array}$ & Definição & Explicação \\
\hline 1 & Importância igual & $\begin{array}{l}\text { Duas ações potenciais contribuem igualmente para } \\
\text { o objetivo }\end{array}$ \\
\hline 3 & $\begin{array}{l}\text { Importância fraca de } \\
\text { uma sobre a outra }\end{array}$ & $\begin{array}{l}\text { A experiência e o julgamento favorecem } \\
\text { levemente uma atividade em relação a outra. }\end{array}$ \\
\hline 5 & Importância forte & $\begin{array}{l}\text { A experiência e o julgamento favorecem } \\
\text { fortemente uma atividade em relação a outra. }\end{array}$ \\
\hline 7 & $\begin{array}{l}\text { Importância muito } \\
\text { forte }\end{array}$ & $\begin{array}{l}\text { Uma atividade é fortemente favorecida em relação } \\
\text { a outra, e sua dominância é demonstrada na } \\
\text { prática. }\end{array}$ \\
\hline 9 & Importância absoluta & $\begin{array}{l}\text { A evidência favorecendo uma atividade em relação } \\
\text { a outra é do mais alto grau de certeza. }\end{array}$ \\
\hline $2,4,6,8$ & $\begin{array}{l}\text { Valores intermediários } \\
\text { entre dois julgamentos } \\
\text { adjacentes }\end{array}$ & $\begin{array}{l}\text { Quando é necessária uma condição de } \\
\text { compromisso. }\end{array}$ \\
\hline Recíprocos & \multicolumn{2}{|c|}{$\begin{array}{l}\text { Se a ação } I \text { tem uma das intensidades de importância ou de preferência de } 1 \\
\text { a } 9 \text { quando comparada com a ação } j \text {, então } j \text { tem o valor recíproco quando } \\
\text { comparado com } i \text {. }\end{array}$} \\
\hline
\end{tabular}

- Acesso (transporte público, instalações de estacionamentos de veículos, custo do estacionamento, disponibilidade de carrinhos de bagagem, disponibilidade de meiofio, disponibilidade de táxi);

- Check-in (tempo de fila no check-in autoatendimento, tempo de fila check-in guichê, eficiência dos funcionários, atendimento/cordialidade funcionários);

- Saguão (facilidade de encontrar o caminho no aeroporto, painéis de informação de voo, distância de caminhada no terminal de passageiros, atendimento/cordialidade dos funcionários, internet/wi-fi, disponibilidade de sanitários, limpeza dos sanitários);

- Sala de embarque (tempo de fila na emigração, atendimento/cordialidade dos funcionários da emigração, conforto na sala de embarque);

- Concessões (instalações de alimentação, valor pago nas instalações de alimentação, disponibilidade de bancos/ caixas eletrônicos/ câmbio, estabelecimentos comerciais, valor pago nos estabelecimentos comerciais).

Já no trabalho de Bandeira (2008), os mesmos cinco critérios (acesso, check-in, saguão, sala de embarque e concessões) foram analisados através de um estudo de caso no Aeroporto Internacional de São Paulo - Governador André Franco Montoro. Neste trabalho foram então estabelecidas prioridades para cada um dos critérios de acordo com uma pesquisa entre os passageiros, estes critérios podem ser vistos na Tabela 2. Como o trabalho de Bandeira (2008) estabeleceu prioridades dos componentes aeroportuários para um aeroporto internacional, e entende-se, da mesma forma que Lupo (2015), que os aeroportos internacionais têm características, requisitos e públicos semelhantes, utilizou-se estes graus de importância para os demais aeroportos internacionais analisados no presente trabalho. Note que a ordem de prioridades dos indicadores, obtida por Bandeira (2008), e apresentada na Tabela 2 são confirmadas no trabalho de Bezerra e Gomes (2015), onde apresenta o check- in como um critério importante na avaliação de satisfação, e "concessões" como o menos influente em relação aos demais indicadores, de acordo com os passageiros.

No questionário elaborado pela SAC os critérios foram analisados através de "pesos" de 1 a 5 , sendo 1 o menor peso possível e 5 o maior peso possível. Dessa forma, cada aeroporto foi avaliado de acordo com os cinco critérios. Porém, para a utilização do método AHP, foi necessária uma conversão dos "pesos" de 1 a 5 utilizados pela SAC para a escala fundamental do método, que vai de 1 a 9 de acordo com o estabelecido por Saaty (1990), e apresentado na Tabela 3 .

Foi necessário fazer dois tipos de conversões para o método AHP. A primeira delas em relação aos critérios (check-in, sala de embarque, acesso, saguão e concessões), onde se têm as prioridades, como apresentado na Tabela 2. Portanto, para construção da matriz de comparação entre prioridades, utilizou-se as equações de 1 a 3 , de forma que o resultado seja compatível com o estabelecido na escala fundamental de Saaty (1990), apresentada na Tabela 3. Desta forma, ao se comparar o critério de check-in com o de concessões (respectivamente $1^{\circ}$ e $5^{\circ}$ na Tabela 2 ) obtémse um valor de 9 na escala fundamental. Já ao comparar o critério de concessões com o de check-in obtém-se o valor recíproco, ou seja, $1 / 9$.

$$
\begin{aligned}
& \text { Se } x<y: \\
& x: y \rightarrow 2(y-x)+1
\end{aligned}
$$

$$
\begin{aligned}
& \text { Se } x>y: \\
& x: y \rightarrow \frac{1}{2(x-y)+1}
\end{aligned}
$$

$$
\begin{gathered}
\text { Se } x=y: \\
x: y=1
\end{gathered}
$$


Tabela 4. Matriz de comparação entre critérios

\begin{tabular}{cccccc}
\hline CRITÉRIO & ACESSO & CHECK-IN & SAGUÃO & SALA DE EMBARQUE & CONCESSÕES \\
\hline ACESSO & 1 & $1 / 5$ & 3 & $1 / 3$ & 5 \\
CHECK-IN & 5 & 1 & 7 & 3 & 9 \\
SAGUÃO & $1 / 3$ & $1 / 7$ & 1 & $1 / 5$ & 3 \\
SALA DE EMBARQUE & 3 & $1 / 3$ & 5 & 1 & 7 \\
CONCESSÕES & $1 / 5$ & $1 / 9$ & $1 / 3$ & $1 / 7$ & 1 \\
\hline
\end{tabular}

Tabela 5. Tabela de conversão do peso da SAC para o peso do método AHP na análise dos aeroportos em relação ao critério acesso.

\begin{tabular}{lcc}
\multicolumn{1}{c}{ Sigla } & \multicolumn{2}{c}{ Acesso } \\
\hline SBBR - Aeroporto de Brasília & SAC & AHP \\
\hline SBCF - Aeroporto de Confins & 3,15167 & 3 \\
SBCT - Aeroporto de Curitiba & 3,79833 & 6 \\
SBCY - Aeroporto de Cuiabá & 4,04 & 7 \\
SBEG - Aeroporto de Manaus & 2,78833 & 1 \\
SBFZ - Aeroporto de Fortaleza & 3,30333 & 4 \\
SBGL - Aeroporto do Rio de Janeiro /Galeão & 3,65333 & 5 \\
SBGR - Aeroporto de Guarulhos & 3,59833 & 5 \\
SBNT - Aeroporto de Natal & 3,28167 & 3 \\
SBPA - Aeroporto de Porto Alegre & 4,43167 & 9 \\
SBRF - Aeroporto do Recife & 3,56167 & 5 \\
SBSV - Aeroporto de Salvador & 3,765 & 6 \\
\hline
\end{tabular}

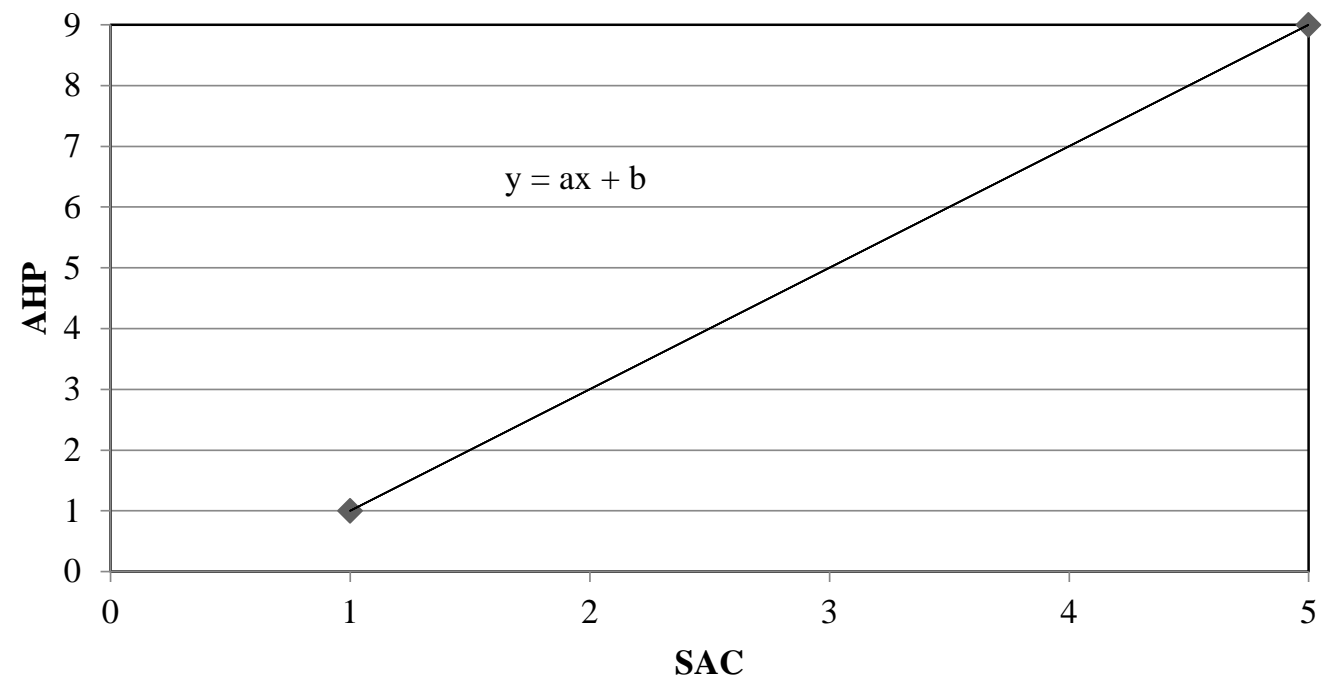

Figura 1. Gráfico genérico das retas de conversão dos pesos da SAC para os pesos do método AHP

em que: x: é a prioridade de um critério;

y: é a prioridade de um segundo critério sendo comparado.

Na Tabela 4 é apresentada a matriz de comparação entre critérios após a aplicação das equações de 1 a 3 conforme descrito anteriormente.

Na comparação entre aeroportos em relação a um determinado critério, houve uma segunda conversão, um exemplo é a Tabela 5, onde a conversão é apresentada para o critério de acesso. Procedeu-se com uma interpolação linear para cada um dos critérios (Figura 1), onde no eixo das ordenadas, têm-se os pesos mínimo e máximo da tabela de Saaty (1990) e no eixo das abcissas, os pesos mínimo e máximo da análise de cada critério do relatório da SAC (2013).

Após a conversão, foi construída a matriz de comparação entre os aeroportos em relação aos critérios, como mostra a Tabela 6. Mas para a comparação entre aeroportos, onde é analisada a relevância de um aeroporto em relação ao outro de acordo com determinado critério, utilizou-se as equações de 4 a 6 . Desta forma, considerando o critério de acesso, ao comparar o aeroporto SBGL com o aeroporto SBNT obtém-se um valor de 1/5. E para a comparação recíproca, obtém-se o valor de 5. Logo, foram feitas cinco matrizes, 12x12, para comparação dos cinco critérios (checkin, sala de embarque, acesso, saguão e concessões).

Se $x<y$ :

$$
\begin{aligned}
& x: y \rightarrow \frac{1}{1+(y-x)} \\
& \text { Se } x>y: \\
& x: y \rightarrow 1+(x-y) \\
& \text { Se } x=y: \\
& x: y=1
\end{aligned}
$$


Tabela 6. Matriz de comparação entre aeroportos em relação ao critério acesso

\begin{tabular}{|c|c|c|c|c|c|c|c|c|c|c|c|c|}
\hline \multirow{2}{*}{ Aeroporto } & \multicolumn{12}{|c|}{ Acesso } \\
\hline & SBBR & $S B C F$ & $S B C T$ & $S B C Y$ & SBEG & SBFZ & SBGL & SBGR & SBNT & SBPA & SBRF & SBSV \\
\hline SBBR & 1 & $1 / 4$ & $1 / 5$ & 3 & $1 / 2$ & $1 / 3$ & $1 / 3$ & 1 & $1 / 7$ & $1 / 3$ & $1 / 4$ & $1 / 3$ \\
\hline SBCF & 4 & 1 & $1 / 2$ & 6 & 3 & 2 & 2 & 4 & $1 / 4$ & 2 & 1 & 2 \\
\hline SBCT & 5 & 2 & 1 & 7 & 4 & 3 & 3 & 5 & $1 / 3$ & 3 & 2 & 3 \\
\hline SBCY & $1 / 3$ & $1 / 6$ & $1 / 7$ & 1 & $1 / 4$ & $1 / 5$ & $1 / 5$ & $1 / 3$ & $1 / 9$ & $1 / 5$ & $1 / 6$ & $1 / 5$ \\
\hline SBEG & 2 & $1 / 3$ & $1 / 4$ & 4 & 1 & $1 / 2$ & $1 / 2$ & 2 & $1 / 6$ & $1 / 2$ & $1 / 3$ & $1 / 2$ \\
\hline SBFZ & 3 & $1 / 2$ & $1 / 3$ & 5 & 2 & 1 & 1 & 3 & $1 / 5$ & 1 & $1 / 2$ & 1 \\
\hline SBGL & 3 & $1 / 2$ & $1 / 3$ & 5 & 2 & 1 & 1 & 3 & $1 / 5$ & 1 & $1 / 2$ & 1 \\
\hline SBGR & 1 & $1 / 4$ & $1 / 5$ & 3 & $1 / 2$ & $1 / 3$ & $1 / 3$ & 1 & $1 / 7$ & $1 / 3$ & $1 / 4$ & $1 / 3$ \\
\hline SBNT & 7 & 4 & 3 & 9 & 6 & 5 & 5 & 7 & 1 & 5 & 4 & 5 \\
\hline SBPA & 3 & $1 / 2$ & $1 / 3$ & 5 & 2 & 1 & 1 & 3 & $1 / 5$ & 1 & $1 / 2$ & 1 \\
\hline SBRF & 4 & 1 & $1 / 2$ & 6 & 3 & 2 & 2 & 4 & $1 / 4$ & 2 & 1 & 2 \\
\hline SBSV & 3 & $1 / 2$ & $1 / 3$ & 5 & 2 & 1 & 1 & 3 & $1 / 5$ & 1 & $1 / 2$ & 1 \\
\hline
\end{tabular}

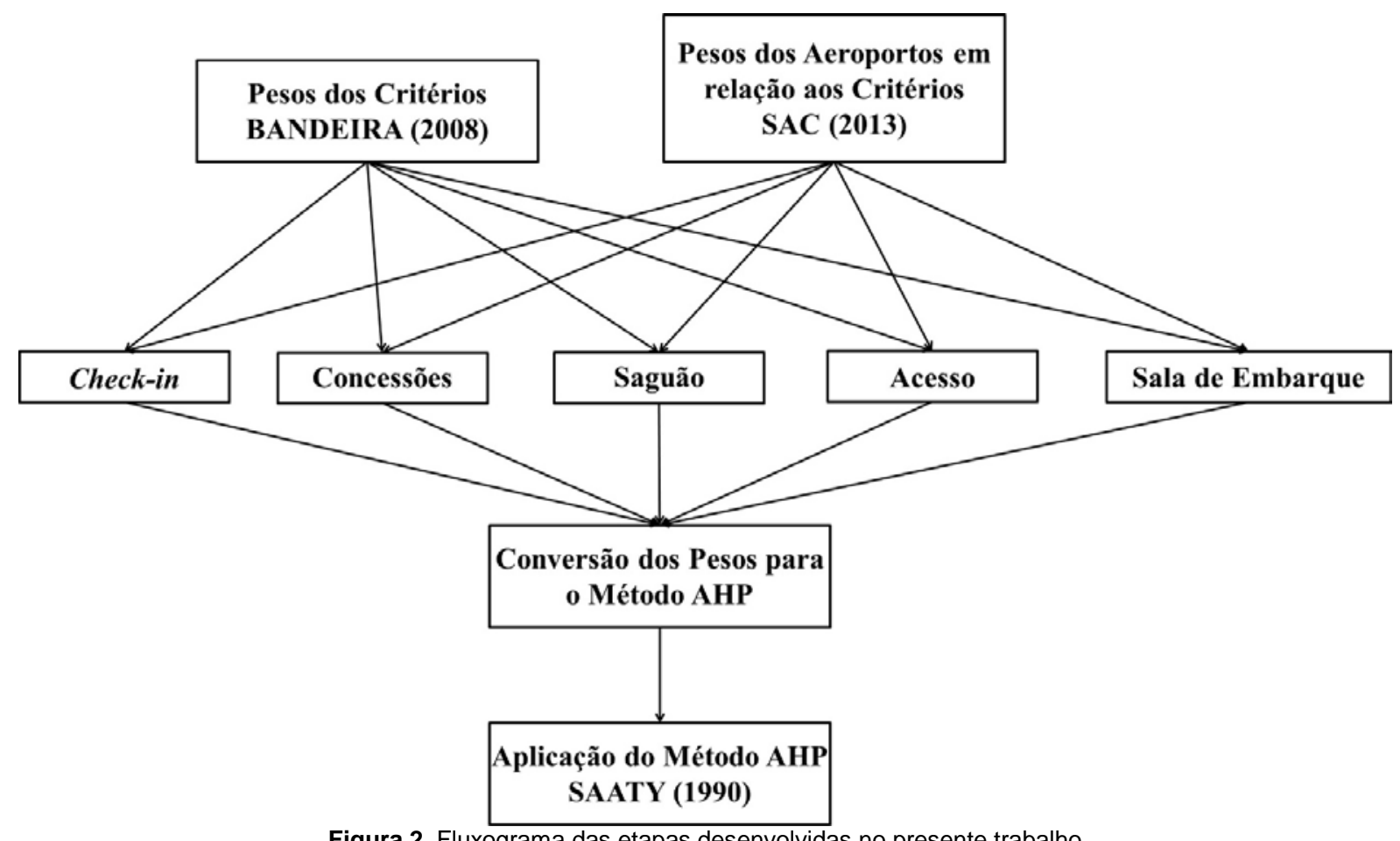

Figura 2. Fluxograma das etapas desenvolvidas no presente trabalho

em que: $x$ : é o peso de um aeroporto de acordo com um critério;

y: é o peso de um segundo aeroporto de acordo com o mesmo critério.

O método AHP baseia-se na escolha de preferências, e essas normalmente são apontadas em entrevistas com especialistas. Porém, de acordo com Saaty (1990), estes podem confundir-se devido à quantidade de critérios analisados, o que resultaria na incoerência das comparações. Por esta razão introduziu-se o cálculo do índice de consistência (Saaty, 1990), que mede o quanto a opinião dada pelo especialista se afasta de uma opinião perfeitamente coerente. Assim, após as comparações (par a par) serem realizadas, é necessário verificar o índice de consistência das matrizes resultantes dessas comparações. Calculou-se então a consistência para as matrizes de comparação apresentadas na Tabela 4 e na Tabela 6, e para as outras quatro matrizes calculadas de forma semelhante a essa última (check-in, sala de embarque, saguão e concessões). Todas as matrizes apresentaram consistência aceitável de acordo com o método AHP. Um resumo de todo desenvolvimento adotado nesse trabalho pode ser visto na Figura 2.

\section{RESULTADOS E DISCUSSÕES}

Com os dados obtidos através do relatório da SAC (2013), os pesos do trabalho de Bandeira (2008) e aplicação do método AHP (Saaty, 1990), pôde-se obter um ranking dos aeroportos em relação aos critérios analisados. No relatório da SAC é apresentada uma classificação geral dos aeroportos pela análise de todos os componentes aeroportuários. Porém, para comparação com os resultados obtidos nesse trabalho, foi necessário fazer uma média aritmética que contempla somente os componentes de infraestrutura analisados pela SAC, obtendo assim uma classificação mais específica. Os resultados obtidos para os critérios de infraestrutura podem ser observados na Tabela 7. Na tabela, os aeroportos listados com as primeiras colocações são aqueles que receberam pesos maiores. Consequentemente, os aeroportos que ficaram com as últimas classificações são aqueles com maiores problemas a serem resolvidos, ou seja, que receberam pesos menores.

Comparando os resultados de classificação apresentados na Tabela 7, podem-se perceber diferenças na ordem listada. Isso pode ser explicado pelo fato do método AHP priorizar os critérios, já no relatório da SAC é considerado 
Tabela 7. Tabela com a ordem de classificação dos aeroportos em relação aos critérios de infraestrutura estudados no presente trabalho

\begin{tabular}{lll}
\hline \multirow{2}{*}{ Aeroporto } & \multicolumn{2}{c}{ Colocação } \\
\cline { 2 - 3 } & AHP & SAC \\
\hline SBCT - Aeroporto de Curitiba & $1^{\circ}$ & $1^{\circ}$ \\
SBNT - Aeroporto de Natal & $2^{\circ}$ & $2^{\circ}$ \\
SBSV - Aeroporto de Salvador & $3^{\circ}$ & $5^{\circ}$ \\
SBCF - Aeroporto de Confins & $4^{\circ}$ & $4^{\circ}$ \\
SBRF - Aeroporto do Recife & $5^{\circ}$ & $3^{\circ}$ \\
SBBR - Aeroporto de Brasília & $6^{\circ}$ & $9^{\circ}$ \\
SBPA - Aeroporto de Porto Alegre & $7^{\circ}$ & $6^{\circ}$ \\
SBGL - Aeroporto do Rio de Janeiro / Galeão & $8^{\circ}$ & $8^{\circ}$ \\
SBFZ - Aeroporto de Fortaleza & $9^{\circ}$ & $7^{\circ}$ \\
SBCY - Aeroporto de Cuiabá & $10^{\circ}$ & $12^{\circ}$ \\
SBGR - Aeroporto de Guarulhos & $11^{\circ}$ & $10^{\circ}$ \\
SBEG - Aeroporto de Manaus & $12^{\circ}$ & $11^{\circ}$ \\
\hline
\end{tabular}

Tabela 8. Tabela com a ordem de classificação geral dos aeroportos pelo relatório da SAC e a comparação da ordem de classificação pelo método AHP

\begin{tabular}{lll}
\hline \multirow{2}{*}{ Aeroporto } & \multicolumn{2}{c}{ Colocação } \\
\cline { 2 - 3 } & AHP & SAC \\
\hline SBCT - Aeroporto de Curitiba & $1^{\circ}$ & $1^{\circ}$ \\
SBNT - Aeroporto de Natal & $2^{\circ}$ & $2^{\circ}$ \\
SBSV - Aeroporto de Salvador & $3^{\circ}$ & $5^{\circ}$ \\
SBCF - Aeroporto de Confins & $4^{\circ}$ & $6^{\circ}$ \\
SBRF - Aeroporto do Recife & $5^{\circ}$ & $4^{\circ}$ \\
SBBR - Aeroporto de Brasília & $6^{\circ}$ & $10^{\circ}$ \\
SBPA - Aeroporto de Porto Alegre & $7^{\circ}$ & $7^{\circ}$ \\
SBGL - Aeroporto do Rio de Janeiro / Galeão & $8^{\circ}$ & $8^{\circ}$ \\
SBFZ - Aeroporto de Fortaleza & $9^{\circ}$ & $3^{\circ}$ \\
SBCY - Aeroporto de Cuiabá & $10^{\circ}$ & $11^{\circ}$ \\
SBGR - Aeroporto de Guarulhos & $11^{\circ}$ & $9^{\circ}$ \\
SBEG - Aeroporto de Manaus & $12^{\circ}$ & $12^{\circ}$ \\
\hline
\end{tabular}

o mesmo peso a todos os itens analisados. Porém, deve-se ressaltar o grande contraste entre as duas análises para o aeroporto de Brasília (SBBR).

O Aeroporto Internacional Juscelino Kubitschek de Brasília, ficou em uma posição mais favorável pelo método AHP do que pelo relatório da SAC. Pela análise feita, o aeroporto recebeu avaliações menores que a média nos critérios acesso e concessões, os quais não têm tanta relevância em comparação com os outros. Porém, recebeu peso máximo (9) para o critério check-in, que tem prioridade máxima de acordo com Bandeira (2008), o que fez sua colocação aumentar.

Como visto na Tabela 7, o aeroporto com maior necessidade de adequações é o Aeroporto Internacional Eduardo Gomes de Manaus, seguido pelo Aeroporto Internacional Governador André Franco Montoro/Guarulhos de São Paulo. O Aeroporto de Manaus recebeu avaliações com peso mínimo (1) nos itens check-in e saguão; já em relação ao acesso e sala de embarque, os pesos foram abaixo da média. Porém, em relação às concessões recebeu avaliação máxima (9), mas esse critério tem a menor prioridade, logo, não o ajudou a subir na ordem de classificação. Em Guarulhos (11 ${ }^{\circ}$ posição) todos os critérios avaliados receberam avaliação abaixo da média.

Em comparação com a classificação geral apresentada pelo relatório da SAC, onde todos os componentes aeroportuários foram analisados (conforme apresentado na Tabela 8), tem-se maior disparidade na ordem de classificação dos aeroportos, principalmente no caso do aeroporto de Fortaleza. O Aeroporto Internacional Pinto Martins de Fortaleza recebeu pior colocação através do método utilizado nesse trabalho do que pelo relatório da SAC, ficando 6 posições atrás. Através do método aqui aplicado, verificou-se que nos critérios check-in e sala de embarque, o aeroporto ficou com pesos abaixo da média, sendo que estes são itens prioritários em relação aos demais critérios. Pela grande diferença de colocações, subentende-se que nos demais critérios analisados pela SAC, os quais este trabalho não contempla (como segurança, aduana, conforto térmico, conforto acústico, limpeza geral, satisfação), o aeroporto se saiu bem ou ao menos acima da média. Sendo assim classificado (pela SAC) como um dos melhores aeroportos dentre os listados.

Para verificar a consistência dos resultados obtidos, calculou-se o índice de consistência de todas as matrizes do método, utilizadas nas etapas de determinação do grau de importância dos critérios. Se o índice de consistência for igual à zero, o sistema será perfeitamente consistente, porém se for maior deverá ser calculado o grau de consistência. Caso o grau de consistência seja considerado insatisfatório (maior que 0,10) há grandes chances da tomada de decisão ser inconsistente.

A Tabela 9 apresenta os graus de consistência calculados para cada uma das matrizes de comparação. Apesar de todos os componentes apresentarem grau de consistência aceitável, deve-se lembrar de que os pesos adotados para os critérios foram tomados de um estudo realizado no Aeroporto Internacional de São Paulo, sendo posteriormente aplicados aos demais aeroportos internacionais aqui em estudo. Assim sendo, seria interessante a realização de um estudo nos demais aeroportos aqui apresentados e a aplicação dos pesos dos componentes aeroportuários de acordo com 
Tabela 9. Tabela com o grau de consistência de cada uma das matrizes de comparação

\begin{tabular}{ll}
\hline $\begin{array}{l}\text { Matriz de } \\
\text { Comparação }\end{array}$ & $\begin{array}{l}\text { Grau de } \\
\text { Consistência }\end{array}$ \\
\hline Critérios & 0,05415333 \\
Acesso & 0,021299291 \\
Check-in & 0,01063289 \\
Saguão & 0,019652779 \\
Sala de Embarque & 0,023944158 \\
Concessões & 0,032334605 \\
\hline
\end{tabular}

o aeroporto real, ao invés de uma simplificação como a adotada nesse trabalho.

O estudo aqui realizado não tem como objetivo sugerir adequações aos sítios aeroportuários, apenas apresentar as fragilidades no sistema dos aeroportos mais importantes do país. Se há mudanças a serem realizadas, apenas um estudo mais específico da necessidade/viabilidade poderá provar. Tanto os resultados apresentados pela SAC quanto os encontrados neste trabalho são interessantes. O primeiro demonstra resultados holísticos do sítio aeroportuário. Já a complementação, com o método AHP aqui utilizada, pôde canalizar as repostas aos critérios prioritários.

\section{CONCLUSÃO}

O estudo contínuo dos níveis de serviços dos aeroportos é importante para garantir o bom atendimento aos clientes. Além disso, a realização de grandes eventos, nos quais há um aumento significativo do fluxo de passageiros merece uma atenção especial, já que se trata de uma situação não corriqueira, além de ter um forte impacto na visão que os estrangeiros têm do país.

Os resultados apontados pelo relatório da SAC são úteis para avaliar quais serviços necessitam de melhorias. Contudo, o resultado está influenciado pelo método adotado, no qual não há diferenciação entre os pesos dos critérios avaliados. Como o valor percebido pelos usuários do aeroporto não é o mesmo para todos os fatores, o método AHP permite levar isto em consideração e fornece resultados distintos ao do relatório. Deste modo, pode-se afirmar que este trabalho procura estar alinhado com a opinião do consumidor, o que ressalta outras nuances do problema em questão. Porém, deve-se ter em mente que, independentemente de qual método é utilizado, é preciso fazer uma análise cuidadosa dos resultados obtidos, a fim de identificar incoerências e particularidades. Este foi o caso do aeroporto de Brasília, que apesar de ter recebido muitas notas abaixo da média, teve sua posição alavancada pela pontuação máxima no critério de maior peso (check-in).

Sabe-se também, tendo sido apontada por Saaty (1990), da dificuldade em realizar comparações com mais de cinco elementos ou critérios. Este estudo propôs comparações par a par, por critério, para 12 aeroportos. Deve-se lembrar de que a avaliação foi tomada previamente através de questionários nos aeroportos (SAC, 2013), não tendo sido, portanto, uma avaliação de experts. Mesmo quando realizada por especialistas, essa análise muitas vezes se apresenta inconsistente pela dificuldade de se comparar um número tão grande de elementos simultâneos (no caso, 12 aeroportos), portanto, os resultados devem ser analisados com cuidado. Além disso, foi feita uma "conversão" de pesos e medidas do relatório da SAC para o método AHP, podendo fragilizar essa ferramenta de tomada de decisão, pois não foi considerada a avaliação consciente dos avaliadores. Apesar dessas limitações, os resultados obtidos no estudo são válidos como referência na identificação de gargalos operacionais nos aeroportos brasileiros, ainda que tenham ocorrido estas "simplificações” metodológicas.

Por fim, vale ressaltar que o presente estudo é capaz apenas de indicar quais são os critérios críticos em que os aeroportos estudados devem focar sua atenção e esforços de melhoria. Para de fato desenvolver projetos para sanar as deficiências identificadas, certamente são necessários estudos mais aprofundados sobre os aspectos específicos que devem ser atacados para garantir a satisfação dos passageiros.

\section{AGRADECIMENTOS}

Os autores gostariam de agradecer a Capes pelo auxílio financeiro.

\section{REFERÊNCIAS}

Bandeira, M. C. G. da S. P. (2008) Análise do nível de serviço em terminais de passageiros aeroportuários. Dissertação. (Mestrado). São José dos Campos: Instituto Tecnológico de Aeronáutica. Disponível em:

$<$ http://www.bdita.bibl.ita.br/tesesdigitais/000552510.pdf>

Bezerra, G. C. L. e C. F. Gomes (2015) The effects of service quality dimensions and passenger characteristics on passenger's overall satisfaction with an airport. Journal of Air Transport Management, v. 44-45, p. 77-81.

DOI: 10.1016/j.jairtraman.2015.03.001.

Borille, G. M. R. e A. R. Correia (2013) A method for evaluating the level of service arrival components at airports. Journal of Air Transport Management, v. 27, p. 5-10.

DOI: 10.1016/j.jairtraman.2012.10.008.

Chen, J. K. C.; A. Batchuluun.; J. Batnasan (2015) Services innovation impact to customer satisfaction and customer value enhancement in airport. Technology in Society, v. 43, p. 219-230. DOI: 10.1016/j.techsoc.2015.05.010.

Correia, A. R.; S. C. Wirasinghe e A. G. de Barros (2008) A global index for level of service evaluation at airport passenger terminals. Transportation Research Part E, v. 44, n. 4, p. 607620. DOI: 10.1016/j.tre.2007.05.009.

Fernandes, E. e R. R. Pacheco (2007) Airport management: a strategic approach. Transportation, v. 34, p. 129-142. DOI: $10.1007 / \mathrm{s} 11116-006-9102-8$.

Lupo, T. (2015) Fuzzy ServPerf model combined with ELECTRE III to comparatively evaluate service quality of international airports in Sicily. Journal of Air Transport Management, v. 42, p. 249-259.

DOI: 10.1016/j.jairtraman.2014.11.006. 
Merket, R. e A. G. Assaf (2015) Using DEA models to jointly estimate service quality perception and profitability - Evidence from international airports. Transportation Research Part A, v. 75, p. 42-50. DOI: 10.1016/j.tra.2015.03.008.

Saaty, T. L. (1990) How to make a decision: the analytic hierarchy process. European Journal of Operational Research, v. 48, p. 9-26. DOI: 10.1016/0377-2217(90)90057-I.

Saaty, T. L. (2008) Decision making with the analytic hierarchy process. Internacional Journal Services Sciences, v. 1, n. 1, p. 83-98. DOI: 10.1504/IJSSCI.2008.017590.

SAC (2013) Relatório Geral dos Indicadores de Desempenho Operacional em Aeroportos (Abril - Junho). Secretaria de Aviação Civil da Presidência da República.

Yoo, K. E. e Y. C. Choi (2006) Analytic hierarchy process approach for identifying relative importance of factors to improve passenger security checks at airports. Journal of Air Transport Management , v. 12, n. 3, p. 135-142.

DOI: 10.1016/j.jairtraman.2005.11.006. 